\title{
Article \\ Neutrophil-to-Lymphocyte Ratio Predicts Development of Immune-Related Adverse Events and Outcomes from Immune Checkpoint Blockade: A Case-Control Study
}

\author{
Pei Yi Lee ${ }^{1,+}$, Kellynn Qi Xuan Oen ${ }^{1,+}$, Grace Rui Si Lim ${ }^{1}{ }^{\mathbb{D}}$, Juanda Leo Hartono ${ }^{1,2}$, Mark Muthiah ${ }^{1,2} \mathbb{D}^{1}$, \\ Daniel Q. Huang ${ }^{1,2} \mathbb{D}^{1}$, Felicia Su Wei Teo ${ }^{1,3}$, Andrew Yunkai Li ${ }^{1,3}$, Anselm Mak ${ }^{1,4}$, Nisha Suyien Chandran ${ }^{1,5}$, \\ Chris Lixian Tan ${ }^{1,5}$, Peiling Yang ${ }^{1,6}$, E Shyong Tai ${ }^{1,6}$, Kay Wei Ping Ng 1,7, Joy Vijayan ${ }^{1,7}$, Yee Cheun Chan ${ }^{1,7}$, \\ Li Ling Tan 1,8, Martin Beng-Huat Lee ${ }^{1,9}$, Horng Ruey Chua ${ }^{1,9}$, Wei Zhen Hong ${ }^{1,9}$, Eng Soo Yap ${ }^{1,10} \oplus$, \\ Dawn K. Lim ${ }^{11,12}$, Yew Sen Yuen ${ }^{11,12}$, Yiong Huak Chan ${ }^{13} \mathbb{1}$, Folefac Aminkeng $\left.{ }^{1} \mathbb{(}\right)$, Alvin Seng Cheong Wong ${ }^{14}$, \\ Yiqing Huang ${ }^{14}$ and Sen Hee Tay $1,4, *$ (i)
}

check for

updates

Citation: Lee, P.Y.; Oen, K.Q.X.; Lim, G.R.S.; Hartono, J.L.; Muthiah, M.; Huang, D.Q.; Teo, F.S.W.; Li, A.Y.; Mak, A.; Chandran, N.S.; et al. Neutrophil-to-Lymphocyte Ratio Predicts Development of

Immune-Related Adverse Events and Outcomes from Immune Checkpoint Blockade: A Case-Control Study. Cancers 2021, 13, 1308. https:// doi.org/10.3390/cancers13061308

Academic Editor:

Constantin Baxevanis

Received: 22 February 2021

Accepted: 10 March 2021

Published: 15 March 2021

Publisher's Note: MDPI stays neutral with regard to jurisdictional claims in published maps and institutional affiliations.

Copyright: (c) 2021 by the authors. Licensee MDPI, Basel, Switzerland. This article is an open access article distributed under the terms and conditions of the Creative Commons Attribution (CC BY) license (https:/ / creativecommons.org/licenses/by/ $4.0 /)$.
1 Department of Medicine, Yong Loo Lin School of Medicine, National University of Singapore, Singapore 119228, Singapore; peiyi.lee@mohh.com.sg (P.Y.L.); kellynn.oen@mohh.com.sg (K.Q.X.O.); e0057008@u.nus.edu (G.R.S.L.); leo_juanda@nuhs.edu.sg (J.L.H.); mark_muthiah@nuhs.edu.sg (M.M.); daniel_huang@nuhs.edu.sg (D.Q.H.); felicia_sw_teo@nuhs.edu.sg (F.S.W.T.); andrew_yunkai_li@nuhs.edu.sg (A.Y.L.); mdcam@nus.edu.sg (A.M.);

nisha_suyien_chandran@nuhs.edu.sg (N.S.C.); chris_lixian_tan@nuhs.edu.sg (C.L.T.);

Peiling_YANG@nuhs.edu.sg (P.Y.); mdctes@nus.edu.sg (ES.T.); Kay_WP_NG@nuhs.edu.sg (K.W.P.N.); joy_vijayan@nuhs.edu.sg (J.V.); Yee_Cheun_CHAN@nuhs.edu.sg (Y.C.C.); li_ling_tan@nuhs.edu.sg (L.L.T.); martin_lee@nuhs.edu.sg (M.B.-H.L.); Horng_Ruey_CHUA@nuhs.edu.sg (H.R.C.); wei_zhen_hong@nuhs.edu.sg (W.Z.H.); Eng_Soo_YAP@nuhs.edu.sg (E.S.Y.); mdcfa@nus.edu.sg (F.A.)

2 Division of Gastroenterology and Hepatology, Department of Medicine, National University Hospital, Singapore 119228, Singapore

3 Division of Respiratory and Critical Care Medicine, Department of Medicine, National University Hospital, Singapore 119228, Singapore

4 Division of Rheumatology, Department of Medicine, National University Hospital, Singapore 119228, Singapore

5 Division of Dermatology, Department of Medicine, National University Hospital, Singapore 119228, Singapore

6 Division of Endocrinology, Department of Medicine, National University Hospital, Singapore 119228, Singapore

7 Division of Neurology, Department of Medicine, National University Hospital, Singapore 119228, Singapore

8 Department of Cardiology, National University Heart Center, National University Hospital, Singapore 119228, Singapore

9 Division of Nephrology, Department of Medicine, National University Hospital, Singapore 119228, Singapore

10 Department of Laboratory Medicine, National University Hospital, Singapore 119228, Singapore

11 Department of Ophthalmology, Yong Loo Lin School of Medicine, National University of Singapore, Singapore 119228, Singapore; dawn_lim@nuhs.edu.sg (D.K.L.); yew_sen_yuen@nuhs.edu.sg (Y.S.Y.)

12 Department of Ophthalmology, National University Hospital, Singapore 119228, Singapore

13 Biostatistics Unit, Yong Loo Lin School of Medicine, National University of Singapore, Singapore 119228, Singapore; medcyh@nus.edu.sg

14 Department of Haematology-Oncology, National University Cancer Institute, National University Hospital, Singapore 119228, Singapore; alvin_sc_wong@nuhs.edu.sg (A.S.C.W.); yiqing_huang@nuhs.edu.sg (Y.H.)

* Correspondence: sen_hee_tay@nuhs.edu.sg

$+\quad$ Pei Yi Lee and Kellynn Qi Xuan Oen contributed equally to the study.

Simple Summary: In this case-control study, we aimed to investigate the relationships between neutrophil-to-lymphocyte ratio (NLR) and platelet-to-lymphocyte ratio (PLR) with the occurrence of immune-related adverse events (irAEs) and clinical outcomes in cancer patients who had received at least one dose of immune checkpoint inhibitor. The study included 91 patients with irAEs and 56 controls. Multiple logistic regression showed that NLR $<3$ at baseline was associated with higher occurrence of irAEs but PLR was not associated with development of irAEs. Multivariate Cox regression showed that development of irAEs and reduction in NLR from baseline to week 6 were associated with longer progression-free survival. Higher NLR values at baseline and/or week 6 were 
independently associated with shorter overall survival (OS). A reduction in NLR from baseline to week 6 was associated with longer OS.

Abstract: The utility of neutrophil-to-lymphocyte ratio (NLR) and platelet-to-lymphocyte ratio (PLR) utility in predicting immune-related adverse events (irAEs) and survival have not been well studied in the context of treatment with immune checkpoint inhibitors (ICIs). We performed a case-control study of cancer patients who received at least one dose of ICI in a tertiary hospital. We examined NLR and PLR in irAE cases and controls. Logistic and Cox regression models were used to identify independent risk factors for irAEs, progression-free survival (PFS), and overall survival (OS). The study included 91 patients with irAEs and 56 controls. Multiple logistic regression showed that $\mathrm{NLR}<3$ at baseline was associated with higher occurrence of irAEs. Multivariate Cox regression showed that development of irAEs and reduction in NLR from baseline to week 6 were associated with longer PFS. Higher NLR values at baseline and/or week 6 were independently associated with shorter OS. A reduction in NLR from baseline to week 6 was associated with longer OS. In this study of cancer patients treated with ICIs, NLR has a bidirectional relationship with adverse outcomes. Lower NLR was associated with increased occurrence of irAEs while higher NLR values were associated with worse clinical outcomes.

Keywords: neutrophil-to-lymphocyte ratio; immunotherapy; immune-related adverse events; survival

\section{Introduction}

Immune checkpoint inhibitors (ICIs) have ushered in a new era in oncology and haematology, with these agents likely to become the backbone of cancer therapy in a wide range of cancer types [1]. However, the success, and therefore use of ICIs has also unveiled the unique challenge they pose-namely their association with immune-related adverse events (irAEs) and in certain cases, resulting in significant morbidity and mortality $[2,3]$. Interestingly, an association between irAEs with treatment response has also been described [4]. Given the significant role that irAEs play in cancer management and may affect long-term patient outcomes, studies have attempted to investigate the role of biomarkers in predicting the occurrence of irAEs. Notably, recent work has also demonstrated that elevated neutrophil-to-lymphocyte ratio (NLR) and platelet-to-lymphocyte ratio (PLR) were associated with poorer outcomes in several malignancies treated with ICIs [5-7]. However, the data on utility of these ratios in predicting irAEs is still emerging. In this case-control study, we aimed to investigate the relationships between NLR and PLR with the occurrence of irAEs and clinical outcomes in cancer patients who had received at least one dose of ICI. In addition, we reviewed the literature of published studies to investigate the association of these ratios with irAE occurrence and efficacy.

\section{Materials and Methods}

\subsection{Patients}

We performed a retrospective case-control study of patients aged 21 years or older treated with at least a single dose of anti-programmed cell death 1 (PD-1)/PD-1 ligand (PD-L1)/cytotoxic T-lymphocyte antigen 4 (CTLA-4)-based ICI at National University Hospital, Singapore from Jun 2014 to Sept 2019. Patients who had received combination ICI-based regimens were excluded. Controls were selected based on stage of cancer, type of cancer and class of ICI used. The study was approved by NHG Domain Specific Review Board B (reference code: 2017/01254) and was carried out in accordance with the principles of the Declaration of Helsinki. All subjects gave written informed consent prior to study inclusion. 


\subsection{Data Collection}

Demographic data including age, gender, race, and clinical data such as Eastern Cooperative Oncology Group (ECOG) Performance Status (PS), body mass index (BMI), smoking status, and brain metastases were collected. Treatment data on the type, duration, and number of cycles of ICI, line of treatment and other cancer treatments, such as concomitant chemotherapy or radiotherapy during ICI were also collected. Patients received the following ICIs until tumor progression, development of unacceptable irAEs, withdrawal or death: nivolumab, pebrolizumab, atezolimumab, avelumab, durvalumab, and tremelimumab. irAEs were graded using the Common Terminology Criteria for Adverse Events v5.0. Patients suspected of having irAEs were reviewed through a chart review and only patients deemed to have irAEs were included as cases. Data was collected for patients with multiple episodes of irAEs, if any. These irAE episodes may be in the same or different organ systems. Progression-free survival (PFS) was calculated from the first day of treatment with ICIs to the date of radiographic or clinical progression or death, whichever came first. Overall survival (OS) was calculated from the first day of treatment with ICIs to the date of death from any cause. Blood counts data at baseline (most recent blood count before ICI initiation), at $6( \pm 2)$ weeks after therapy initiation, and at irAE diagnosis were used to calculate NLR (absolute neutrophil count/absolute lymphocyte count) and PLR (platelet count/absolute lymphocyte count). Patients with ongoing sepsis were excluded. All data were checked by the primary investigator (S.H.T.). Two other investigators (A.S.C.W and Y.H.) resolved any differences in the interpretation. We used PubMed to identify English studies that reported the association between NLR and PLR with irAE occurrence and efficacy of ICIs from database inception to August 2020.

\subsection{Statistical Analysis}

The primary outcome was to evaluate the association of NLR and PLR with the development of irAEs in this case-control study. Secondary outcomes were to evaluate the prognostic values of NLR and PLR on clinical outcomes such as PFS and OS. NLR data were analyzed as a continuous variable or dichotomized into prespecified cutoffs for $\geq 3$ versus $<3$ and $\geq 5$ versus $<5$ [7]. PLR data were analyzed as a continuous variable or dichotomized into prespecified cutoffs for $\geq 180$ versus $<180$ [7]. Normal distribution was assessed using the Shapiro-Wilk test. Continuous and categorical data were analyzed using the Mann-Whitney U test and Pearson's chi-square test, respectively. The association between NLR and PLR and the development of irAEs was analyzed by univariate logistic regression. Variables that trended toward a significant association $(p<0.1)$ were further evaluated with multivariate analysis. When performing survival analyses, the dataset was considered as a whole. PFS and OS were calculated using the Kaplan-Meier method. Similarly, univariate followed by multivariate Cox regression models were employed to find independent predictors associated with PFS and OS. Statistical significance was defined as a two-tailed $\mathrm{p}$ value of $<0.05$. All statistical analyses were performed with SPSS, version 26 (IBM Corp, Armonk, NY, USA).

\section{Results}

\subsection{Patient Characteristics}

Table 1 shows the comparison of characteristics between patients who experienced irAEs and those who did not. A total of 147 patients were included in the study, of which $91(61.9 \%)$ were irAE cases and $56(38.1 \%)$ were controls. The median age was 62.0 years (54.0-69.0) and the median time on follow-up was 10.2 months (3.7-19.6). The primary malignancies in this study were lung cancer (55.4\%), colorectal cancer $(7.4 \%)$, nasopharyngeal carcinoma (6.1\%), gastric cancer (4.1\%), hepatocellular carcinoma ( $4.1 \%)$, and others. The majority of patients had ECOG PS of 0-1 (87.2\%). The irAE cases and controls had similar demographic, clinical and treatment histories. The median neutrophil, lymphocyte and platelet counts at baseline were similar in both groups. NLR and PLR at baseline and week 6 were not significantly different in both groups, although week 6 NLR 
trended higher in controls compared to irAE cases $(p=0.063)$. Endocrinopathies were the most common irAEs $(8.1 \%)$, followed by hepatic $(7.4 \%)$ and neurological $(7.4 \%)$ disorders. Some patients developed multiple episodes of irAEs: 2 episodes in 32 (21.6\%); 3 episodes in $15(10.1 \%)$, and 4 episodes in $4(2.7 \%)$.

Table 1. Comparison of characteristics of patients with or without immune-related adverse events (irAEs). Data are frequency (\%) or median (interquartile range).

\begin{tabular}{|c|c|c|c|}
\hline & $\begin{array}{l}\text { Patients with irAEs } \\
\quad(n=91)\end{array}$ & $\begin{array}{l}\text { Patients without } \\
\text { irAEs }(n=56)\end{array}$ & $p$-Value \\
\hline Age (years) & $61.0(52.0-70.0)$ & $63.0(55.5-68.0)$ & 0.500 \\
\hline Gender (female) & $31(34.1)$ & $17(30.4)$ & 0.641 \\
\hline Ethnicity & & & 0.165 \\
\hline Chinese & $67(73.6)$ & $44(78.6)$ & \\
\hline Malay & $11(12.1)$ & $2(3.6)$ & \\
\hline Indian & $2(2.2)$ & $4(7.1)$ & \\
\hline Others & $11(12.1)$ & $6(10.7)$ & \\
\hline ECOG PS & & & 0.949 \\
\hline 0 & $30(33.0)$ & $19(33.9)$ & \\
\hline 1 & $48(52.7)$ & $31(55.4)$ & \\
\hline 2 & $2(5.5)$ & $4(7.1)$ & \\
\hline Smoking status & & & 0.486 \\
\hline Smoker & $40(44.0)$ & $23(41.1)$ & \\
\hline Non-smoker & $35(38.5)$ & $26(46.4)$ & \\
\hline BMI & $22.4(19.2-25.0)$ & $22.2(20.6-23.5)$ & 0.945 \\
\hline Cancer stage & & & 0.380 \\
\hline I & $6(6.6)$ & $1(1.8)$ & \\
\hline II & $6(6.6)$ & $2(3.6)$ & \\
\hline III & $13(14.3)$ & $9(16.1)$ & \\
\hline IV & $54(59.3)$ & $41(73.2)$ & \\
\hline Cancer type & & & 0.803 \\
\hline Lung cancer & $44(48.4)$ & $37(66.1)$ & \\
\hline Renal cell carcinoma & $1(1.1)$ & $1(1.8)$ & \\
\hline Nasopharyngeal carcinoma & $6(6.6)$ & $3(5.4)$ & \\
\hline Melanoma & $1(1.1)$ & $1(1.8)$ & \\
\hline $\begin{array}{l}\text { Duration of ICI treatment } \\
\text { (days) }\end{array}$ & $72.5(28.0-215.5)$ & $68.0(21.0-143.0)$ & 0.225 \\
\hline No. of cycles & $4(2-11)$ & $4(2-8)$ & 0.166 \\
\hline Class of ICI treatment & & & 0.452 \\
\hline Anti-CTLA-4 & $2(2.2)$ & $0(0.0)$ & \\
\hline Anti-PD-1 & $54(59.3)$ & $37(66.1)$ & \\
\hline Anti-PD-L1 & $34(37.4)$ & $19(33.9)$ & \\
\hline Line of treatment & & & 0.946 \\
\hline
\end{tabular}


Table 1. Cont.

\begin{tabular}{|c|c|c|c|}
\hline & $\begin{array}{l}\text { Patients with irAEs } \\
\qquad(n=91)\end{array}$ & $\begin{array}{l}\text { Patients without } \\
\text { irAEs }(n=56)\end{array}$ & $p$-Value \\
\hline 1 st & $25(27.5)$ & $15(26.8)$ & \\
\hline 2nd & $25(27.5)$ & $18(32.1)$ & \\
\hline $3 r d$ & $18(19.8)$ & $10(17.9)$ & \\
\hline 4th and beyond & $21(23.1)$ & $12(21.4)$ & \\
\hline Concomitant chemotherapy & $11(12.1)$ & $8(14.3)$ & 0.700 \\
\hline Concomitant radiotherapy & $9(9.9)$ & $5(8.9)$ & 0.847 \\
\hline Brain metastases & $9(9.9)$ & $8(14.3)$ & 0.432 \\
\hline \multicolumn{4}{|l|}{$\begin{array}{l}\text { Baseline FBC data } \\
\qquad\left(\mathrm{N} \times 10^{9} / \mathrm{L}\right)\end{array}$} \\
\hline Neutrophil & $4.29(3.23-5.73)$ & $5.49(3.40-7.23)$ & 0.122 \\
\hline Lymphocyte & $1.30(0.88-1.74)$ & $1.25(0.82-1.77)$ & 0.603 \\
\hline Platelet & $264(195-342)$ & $305(213-367)$ & 0.177 \\
\hline \multicolumn{4}{|l|}{ NLR } \\
\hline Baseline & $3.12(2.22-5.93)$ & $3.77(2.92-7.49)$ & 0.136 \\
\hline Week 6 & $3.20(2.23-5.08)$ & $4.21(2.48-6.83)$ & 0.063 \\
\hline \multicolumn{4}{|l|}{ PLR } \\
\hline Baseline & $211.84(131.72-317.09)$ & $213.00(144.53-384.15)$ & 0.582 \\
\hline Week 6 & $196.00(134.97-337.79)$ & $236.97(172.62-383.85)$ & 0.116 \\
\hline \multicolumn{4}{|l|}{$\begin{array}{l}\text { Organ system affected at 1st } \\
\text { irAE }\end{array}$} \\
\hline Endocrine & $12(8.1)$ & & \\
\hline Hepatic & $11(7.4)$ & & \\
\hline Neurological & $11(7.4)$ & & \\
\hline Gastrointestinal & $9(6.1)$ & & \\
\hline Dermatological & $8(5.4)$ & & \\
\hline Pulmonary & $5(3.4)$ & & \\
\hline Rheumatic & $3(2.0)$ & & \\
\hline
\end{tabular}

Abbreviations: ECOG PS, Eastern Cooperative Oncology Group Performance Status; BMI, body mass index CTLA-4, cytotoxic T-lymphocyte antigen 4; PD-1, programmed cell death 1; PD-L1, PD-1 ligand; FBC, full blood count; NLR, neutrophil-to-lymphocyte ratio; PLR, platelet-to-lymphocyte ratio.

\subsection{Prognostic Role of NLR and PLR for the Development of irAEs}

Univariate logistic regression was performed to assess the risk factors for irAEs (Table $\mathrm{S1})$. Interestingly, patients with lung cancer, compared to other cancers, were less likely to develop irAEs (odds ratio (OR) $=0.49,95 \%$ confidence interval $(\mathrm{CI}) 0.24-0.98, p=0.044$ ). Using the ratio data, baseline NLR $<3(\mathrm{OR}=2.50,95 \%$ CI 1.20-5.22, $p=0.015)$ was a risk factor for irAEs. Subsequent multivariate analysis revealed baseline NLR $<3$ (adjusted $\mathrm{OR}=2.27,95 \%$ CI 1.07-4.82, $p=0.034$ ) remained statistically significant in predicting development of irAEs (Table S2). However, the median NLR was not significantly different for Grade $1-2$ irAEs versus Grade $3-4$ irAE (4.57 vs. 4.04, $p=0.638$ ) at the first irAE episode, though numerically lower in higher grades of irAEs (Figure 1). PLR was not associated with development of irAEs. 


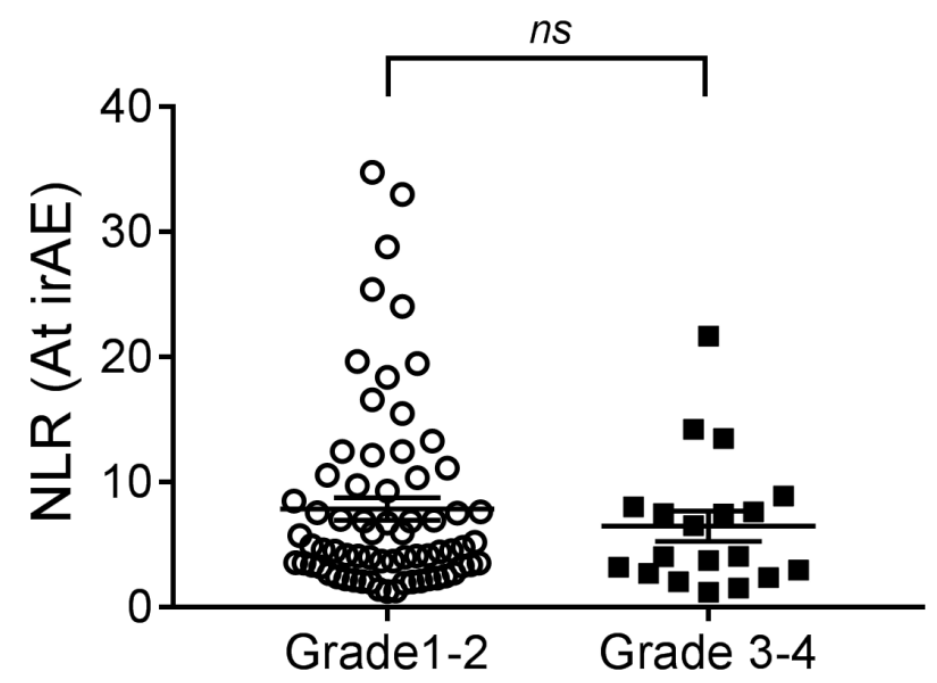

Figure 1. Comparison of neutrophil-to-lymphocyte ratio (NLR) between Grade 1-2 immune-related adverse event (irAE) and Grade 3-4 irAE at the first irAE episode. NLR was numerically lower in patients with higher grades of irAEs. The horizontal line represents the mean NLR and the standard error of the mean.

\subsection{Univariate Analyses for PFS and OS}

The median PFS and OS were 3.0 months (Q1:Q3 2.3; 3.7) and 10.6 months (Q1:Q3 7.5:13.8), respectively. According to the univariate analyses for PFS and OS, no significant differences were found with respect to age, gender, cancer stage, cancer type and class of ICI treatment. However, PFS and OS were reduced in patients with ECOG PS 2 but increased in patients on concomitant chemotherapy (Table S3). PFS was shorter in patients with higher baseline NLR, baseline PLR, week 6 NLR, week 6 NLR $\geq 3$, and week 6 NLR $\geq 5$ (Table S3). Reduction in PLR and NLR from baseline to week 6 was associated with longer PFS (Table S3). In the univariate analysis for OS, development of irAEs (HR 0.58, 95\% CI 0.39-0.86, $p=0.006$ ), higher BMI and reduction in NLR from baseline to week 6 were associated with longer OS (Table S3). OS was shorter in patients with higher baseline NLR, baseline NLR $\geq 3$, baseline NLR $\geq 5$, week 6 NLR, week 6 NLR $\geq 3$, week 6 NLR $\geq 5$, baseline PLR, baseline PLR $\geq 180$, and week 6 PLR (Table S3).

\subsection{Multivariate Analyses for PFS}

Multivariable analyses demonstrated that development of irAEs (adjusted HR 0.67, 95\% CI 0.45-0.99, $p=0.046$ ) and reduction of NLR from baseline to week 6 (adjusted HR 0.56, $95 \%$ CI $0.33-0.95, p=0.031$ ) were independent prognostic factors for longer PFS (Table 2).

Table 2. Multivariate analyses of progression-free survival (PFS).

\begin{tabular}{cccc}
\hline Variable & Category & PFS & \\
\hline & & Adjusted HR (95\% CI) & $p$ Value \\
\hline Model 1 & & & \\
\hline ECOG PS & 2 & $1.18(0.49-2.83)$ & 0.718 \\
\hline irAE status & irAE case & $0.67(0.45-0.99)$ & 0.046 \\
\hline No. of cycles & & $0.91(0.88-0.94)$ & $<0.001$ \\
\hline Concomitant chemotherapy & & $0.61(0.33-1.13)$ & 0.115 \\
\hline
\end{tabular}


Table 2. Cont.

\begin{tabular}{|c|c|c|c|}
\hline Variable & Category & PFS & \\
\hline & & Adjusted HR (95\% CI) & $p$ Value \\
\hline Baseline NLR & & $1.03(0.98-1.09)$ & 0.221 \\
\hline Baseline PLR & & $1.00(1.00-1.00)$ & 0.764 \\
\hline \multicolumn{4}{|l|}{ Model 2* } \\
\hline ECOG PS & 2 & $1.10(0.37-3.24)$ & 0.862 \\
\hline irAE status & irAE case & $0.77(0.50-1.19)$ & 0.240 \\
\hline No. of cycles & & $0.91(0.88-0.95)$ & $<0.001$ \\
\hline Concomitant chemotherapy & & $0.83(0.45-1.53)$ & 0.546 \\
\hline Week 6 NLR & & $1.05(1.00-1.11)$ & 0.066 \\
\hline Reduction in PLR & $\begin{array}{c}\text { Baseline/Week } 6 \\
\text { PLR ratio } \geq 1\end{array}$ & $0.64(0.40-1.04)$ & 0.071 \\
\hline \multicolumn{4}{|l|}{ Model 3 * } \\
\hline ECOG PS & 2 & $1.10(0.37-3.28)$ & 0.865 \\
\hline irAE status & irAE case & $0.76(0.49-1.18)$ & 0.226 \\
\hline No. of cycles & & $0.91(0.88-0.94)$ & $<0.001$ \\
\hline Concomitant chemotherapy & & $0.80(0.43-1.49)$ & 0.485 \\
\hline Week 6 NLR & $\mathrm{NLR} \geq 3$ & $1.27(0.80-2.00)$ & 0.307 \\
\hline Reduction in PLR & $\begin{array}{c}\text { Baseline/Week } 6 \\
\text { PLR ratio } \geq 1\end{array}$ & $0.60(0.37-0.95)$ & 0.029 \\
\hline \multicolumn{4}{|l|}{ Model $4 *$} \\
\hline ECOG Performance & 2 & $1.23(0.42-3.61)$ & 0.705 \\
\hline irAE status & irAE case & $0.77(0.49-1.18)$ & 0.229 \\
\hline No. of cycles & & $0.91(0.88-0.95)$ & $<0.001$ \\
\hline Concomitant chemotherapy & & $0.77(0.42-1.42)$ & 0.399 \\
\hline Week 6 NLR & NLR $\geq 5$ & $1.17(0.73-1.8)$ & 0.521 \\
\hline Reduction in PLR & $\begin{array}{c}\text { Baseline/Week } 6 \\
\text { PLR ratio } \geq 1\end{array}$ & $0.59(0.37-0.95)$ & 0.031 \\
\hline \multicolumn{4}{|l|}{ Model 5* } \\
\hline ECOG PS & 2 & $1.37(0.46-4.03)$ & 0.574 \\
\hline irAE status & irAE case & $0.72(0.47-1.11)$ & 0.141 \\
\hline No. of cycles & & $0.92(0.88-0.95)$ & $<0.001$ \\
\hline Concomitant chemotherapy & & $0.93(0.49-1.76)$ & 0.811 \\
\hline Reduction in NLR & $\begin{array}{c}\text { Baseline/Week } 6 \\
\text { NLR ratio } \geq 1\end{array}$ & $0.56(0.33-0.95)$ & 0.031 \\
\hline Reduction in PLR & $\begin{array}{l}\text { Baseline/Week } 6 \\
\text { PLR ratio } \geq 1\end{array}$ & $0.73(0.44-1.22)$ & 0.228 \\
\hline
\end{tabular}

* Patients on follow-up for at least 6 weeks included in the model. Abbreviations: PFS, progression-free survival; irAE, immune-related adverse event.

Figure 2 illustrates the adjusted survival curves for PFS according to irAE status. Patients with irAEs had significantly longer PFS after ICI treatment compared to controls. Patients with reduction in NLR after ICI therapy had longer PFS (6.0 vs. 2.0 months, $p<0.001)$. 


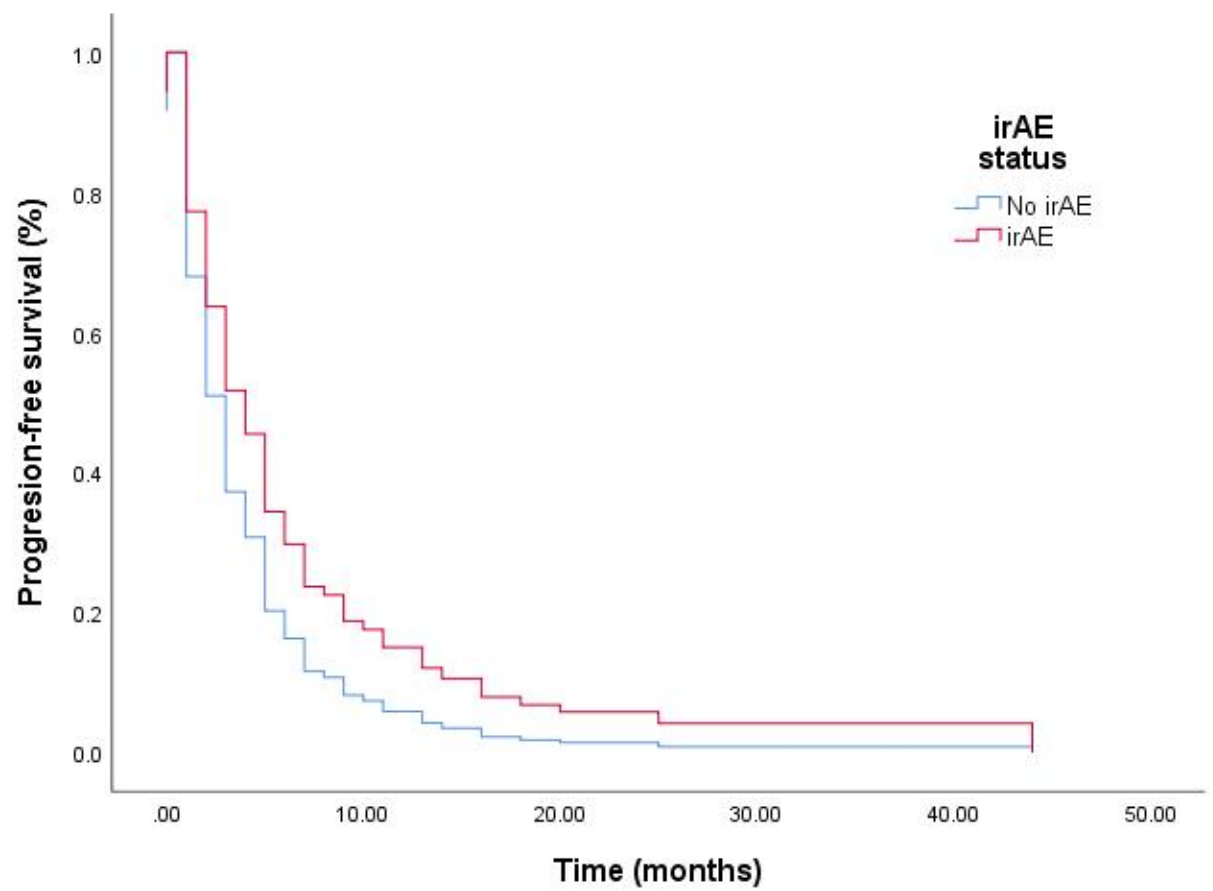

Figure 2. Adjusted survival curves for progression-free survival (PFS) according to irAE status. Comparison of survival curves in patients with or without irAE(s) for PFS.

\subsection{Multivariate Analyses for OS}

In the multivariate Cox regression analyses, the NLR variables such as baseline NLR (adjusted HR 1.04, 95\% CI 1.02-1.06, $p=0.001$ ), baseline NLR $\geq 3$ (adjusted HR 2.64, 95\% CI 1.49-4.69, $p=0.001$ ), week 6 NLR (adjusted HR 1.29, 95\% CI 1.17-1.42, $p<0.001$ ), week 6 NLR $\geq 3$ (adjusted HR 3.12, 95\% CI 1.73-5.61, $p<0.001$ ), and week 6 NLR $\geq 5$ (adjusted HR $3.55,95 \%$ CI 1.84-6.85, $p<0.001$ ) were independently associated with shorter OS (Table 3). Higher BMI and reduction of NLR from baseline to week 6 (adjusted HR 0.38, 95\% CI $0.20-0.62, p<0.001$ ) were associated with longer OS (Table 3). Patients with reduction in NLR after ICI therapy had longer OS (18.2 vs. 6.4 months, $p<0.001)$.

Table 3. Multivariate analyses of overall survival (OS).

\begin{tabular}{cccc}
\hline Variable & Category & OS & \\
\hline & & Adjusted HR (95\% CI) & $p$ Value \\
\hline Model 1 & & & \\
\hline ECOG PS & 2 & $2.09(0.82-5.36)$ & 0.124 \\
\hline BMI & & $0.92(0.86-0.97)$ & 0.004 \\
\hline Cancer type & Lung cancer & $0.78(0.50-1.21)$ & 0.268 \\
\hline irAE status & irAE case & $0.68(0.44-1.05)$ & 0.084 \\
\hline No. of cycles & & $0.88(0.84-0.93)$ & $<0.001$ \\
\hline Concomitant chemotherapy & & $0.52(0.26-1.05)$ & 0.066 \\
\hline Baseline NLR & & $1.04(1.02-1.06)$ & 0.001 \\
\hline Baseline PLR & PLR $\geq 180$ & $1.12(0.70-1.81)$ & 0.631 \\
\hline Model 2 & & &
\end{tabular}


Table 3. Cont.

\begin{tabular}{|c|c|c|c|}
\hline Variable & Category & OS & \\
\hline & & Adjusted HR (95\% CI) & $p$ Value \\
\hline ECOG PS & 2 & $2.37(0.96-5.87)$ & 0.061 \\
\hline BMI & & $0.90(0.85-0.95)$ & $<0.001$ \\
\hline Cancer type & Lung cancer & $0.71(0.46-1.10)$ & 0.122 \\
\hline irAE status & irAE case & $0.75(0.48-1.16)$ & 0.199 \\
\hline No. of cycles & & $0.86(0.82-0.91)$ & $<0.001$ \\
\hline Concomitant chemotherapy & & $0.64(0.32-1.26)$ & 0.196 \\
\hline Baseline NLR & $N L R \geq 3$ & $2.64(1.49-4.69)$ & 0.001 \\
\hline Baseline PLR & PLR $\geq 180$ & $0.72(0.41-1.27)$ & 0.257 \\
\hline \multicolumn{4}{|l|}{ Model 3} \\
\hline ECOG PS & 2 & $2.48(0.96-6.39)$ & 0.060 \\
\hline BMI & & $0.91(0.86-0.97)$ & 0.003 \\
\hline Cancer type & Lung cancer & $0.75(0.49-1.17)$ & 0.207 \\
\hline irAE status & irAE case & $0.67(0.43-1.04)$ & 0.074 \\
\hline No. of cycles & & $0.88(0.84-0.93)$ & $<0.001$ \\
\hline Concomitant chemotherapy & & $0.56(0.28-1.12)$ & 0.102 \\
\hline Baseline NLR & $N L R \geq 5$ & $1.46(0.87-2.43)$ & 0.149 \\
\hline Baseline PLR & PLR $\geq 180$ & $1.12(0.68-1.84)$ & 0.666 \\
\hline \multicolumn{4}{|l|}{ Model $4^{*}$} \\
\hline ECOG PS & 2 & $1.45(0.46-4.61)$ & 0.527 \\
\hline BMI & & $0.90(0.84-0.97)$ & 0.004 \\
\hline Cancer type & Lung cancer & $0.94(0.58-1.51)$ & 0.791 \\
\hline irAE status & irAE case & $0.72(0.45-1.16)$ & 0.178 \\
\hline No. of cycles & & $0.92(0.88-0.95)$ & $<0.001$ \\
\hline Concomitant chemotherapy & & $1.18(0.56-2.49)$ & 0.663 \\
\hline Week 6 NLR & & $1.29(1.17-1.42)$ & $<0.001$ \\
\hline Week 6 PLR & & $1.00(1.00-1.00)$ & 0.006 \\
\hline \multicolumn{4}{|l|}{ Model $5 *$} \\
\hline ECOG PS & 2 & $2.23(0.74-6.72)$ & 0.156 \\
\hline BMI & & $0.91(0.85-0.97)$ & 0.006 \\
\hline Cancer type & Lung cancer & $0.72(0.44-1.18)$ & 0.197 \\
\hline irAE status & irAE case & $0.67(0.42-1.08)$ & 0.101 \\
\hline No. of cycles & & $0.89(0.86-0.93)$ & $<0.001$ \\
\hline Concomitant chemotherapy & & $1.00(0.47-2.12)$ & 0.997 \\
\hline Week 6 NLR & $\mathrm{NLR} \geq 3$ & $3.12(1.73-5.61)$ & $<0.001$ \\
\hline Week 6 PLR & & $1.00(1.00-1.00)$ & 0.823 \\
\hline \multicolumn{4}{|l|}{ Model 6* } \\
\hline ECOG PS & 2 & $2.81(0.91-8.69)$ & 0.074 \\
\hline
\end{tabular}


Table 3. Cont.

\begin{tabular}{|c|c|c|c|}
\hline Variable & Category & OS & \\
\hline & & Adjusted HR (95\% CI) & $p$ Value \\
\hline BMI & & $0.93(0.86-1.00)$ & 0.035 \\
\hline Cancer type & Lung cancer & $0.85(0.53-1.38)$ & 0.517 \\
\hline irAE status & irAE case & $0.69(0.43-1.10)$ & 0.117 \\
\hline No. of cycles & & $0.91(0.87-0.95)$ & $<0.001$ \\
\hline Concomitant chemotherapy & & $0.84(0.41-1.72)$ & 0.631 \\
\hline Week 6 NLR & $\mathrm{NLR} \geq 5$ & $3.55(1.84-6.85)$ & $<0.001$ \\
\hline Week 6 PLR & & $1.00(1.00-1.00)$ & 0.395 \\
\hline \multicolumn{4}{|l|}{ Model $7^{*}$} \\
\hline ECOG PS & 2 & $4.06(1.26-13.05)$ & 0.019 \\
\hline BMI & & $0.94(0.87-1.01)$ & 0.071 \\
\hline Cancer type & Lung cancer & $1.08(0.68-1.73)$ & 0.735 \\
\hline irAE status & irAE case & $0.64(0.40-1.02)$ & 0.063 \\
\hline No. of cycles & & $0.90(0.86-0.95)$ & $<0.001$ \\
\hline Concomitant chemotherapy & & $1.20(0.56-2.59)$ & 0.637 \\
\hline Reduction in NLR & $\begin{array}{l}\text { Baseline/Week } 6 \\
\text { NLR ratio } \geq 1\end{array}$ & $0.38(0.20-0.62)$ & $<0.001$ \\
\hline Week 6 PLR & & $1.00(1.00-1.00)$ & 0.222 \\
\hline
\end{tabular}

* Patients on follow-up for at least 6 weeks included in the model. Abbreviation: OS, overall survival.

\section{Discussion}

Over the past decade, ICIs were introduced to the field of oncology and have since shown great clinical efficacy in reducing the disease burden in cancer patients [8]. As ICIs are increasingly employed in oncological treatment, it is important to look at the possible adverse events and overall outcomes associated with its use. NLR and PLR are simple and inexpensive biomarkers that can be obtained from basic laboratory tests commonly done in standard clinical practice. We focused on the NLR and PLR from the blood counts data as both biomarkers have been extensively studied in autoimmune diseases [9]. For example, the monocyte-to-lymphocyte ratio has not been described in association with systemic lupus erythematosus. In the present retrospective case-control study, we showed that baseline, week 6 and/or post-treatment reduction in NLR were effective in predicting complications (development of irAEs) and survival (PFS and OS). Our study also showed that patients who developed irAEs had longer PFS compared to those who did not. This finding is in keeping with a recent meta-analysis involving 4971 patients with multiple cancer types [10].

Neutrophil-to-lymphocyte ratio was first described by Zahorec et al. in 2001 and PLR was later described by Smith et al. in 2008 [11,12]. It is often asked if there is a biological relevance to these ratios. It is reasonable to speculate that the NLR is a measure of polymorphonuclear myeloid-derived suppressor cells (PMN-MDSCs) released from the bone marrow as a result of chronic inflammation [13]. The hallmark of PMNMDSCs is their ability to suppress T lymphocyte function [14]. The most prominent factors implicated in MDSC suppressive activity include increased production of ROS and nitric oxide, upregulation of arginase I and production of prostaglandin E2 [15,16]. Importantly, Basu et al. reported a significant correlation between NLR and MDSCs recently [17]. We hypothesize that the biological activity of PMN-MDSCs suppressing T lymphocytes, expressed in the NLR, could shape the balance between autoimmunity and cancer. With regards to the PLR, the magnitude of stress-induced hypercortisolemia with subsequent release of platelets from the bone marrow and cortisol-induced lymphopenia result in the 
elevation of PLR in inflammatory disease states [18]. However, the PLR has not yet been ascribed to reflect the activity of any biological cell type.

Our study found that baseline NLR $<3$ was a significant predictor for developing irAEs. Similar findings were noted in previously published cross-sectional studies, where reduced baseline NLR was found to be a significant risk factor for the development of irAEs (Table S4) [7,19-21]. Though the mechanism of irAEs is unclear, this finding is consistent with the hypothesis that NLR is a measure of PMN-MDSCs suppressing the non-specific inflammation and autoimmune response mediated by $\mathrm{T}$ lymphocytes due to the effect of ICIs [17,22]. Conversely, studies by Owen et al. and Peng et al. were not supportive of our findings (Table S4) [6,23]. Owen et al. noted no significant association between baseline NLR and irAEs, while Peng et al. noted that baseline NLR $<5$ was associated with paradoxical lower risk of irAEs $[6,23]$. A majority of the patients in the study by Owen et al. were treated with nivolumab, and hence their results may not be fully applicable to patients treated with other ICIs. Another possible reason for this variation in findings could be attributed to the effects of genetics, age, gender, BMI, and other lifestyle or environmental factors on NLR and PLR, that may not have been fully taken into consideration in previous studies [24]. Association between low PLR and development of irAEs was not observed in our study. This is somewhat surprising, as it has been noted that the PLR is also an inflammatory marker in autoimmune conditions such as antineutrophil cytoplasmic antibody-associated vasculitis, Beçhet disease, rheumatoid arthritis, and systemic lupus erythematosus [18]. However, as discussed above, PLR may not be a surrogate marker of MDSCs like NLR and suppress autoreactive T lymphocyte function.

Elevated NLR and PLR have been shown to be significantly associated with worse outcomes in malignancies [25-27]. Specifically, similar findings have been reported in cancer patients who received ICI therapy (Table S5) $[5,20,23,28-30]$. In line with the literature, our study also noted that elevated NLR, and to a much lesser extent PLR, were associated with shorter OS and PFS in cancer patients who received at least one dose of ICI. However, as only 2 patients received anti-CTLA-4, the presented data should apply only to anti-PD1/PD-L1-based ICI. Biologically, the NLR can potentially reflect the balance of the immune system in the context of a malignancy. Controversy surrounds neutrophil function in cancer because neutrophils have been shown to possess both pro- and anti-tumor properties [31]. With tumor progression, dynamic changes in neutrophil composition result in a switch from an overall anti- to pro-tumor neutrophil contribution [31]. An increasing number of studies have linked increased peripheral blood neutrophils or NLR to tumor-infiltrating neutrophils. Gentles et al. analyzed cancer gene expression and clinical outcome data for approximately 18,000 patients and 39 distinct malignancies [32]. Of the tumor-associated leukocytes, neutrophil signature emerged as the most adverse cancer-wide prognostic population [32]. Lymphocytes are effective suppressors of cancer progression and their presence, particularly in the tumor microenvironment, is thought to reflect host immunity [33]. Another interesting finding in our analysis was the reduction in NLR, but not PLR, after ICI therapy was associated with significantly improved OS. Hence, NLR, unlike PLR, could also be a measure of PMN-MDSCs suppressing anti-tumor T lymphocyte responses in the tumor microenvironment. Of interest, anti-CTLA-4 treatment has been demonstrated to decrease PMN-MDSCs in patients with metastatic melanoma [34]. Therefore, the ability of NLR to reflect PMN-MDSC activity in anti-CTLA-4-based therapy needs to be examined.

The current study has several limitations and strengths. Our study is mainly limited by its retrospective nature and relatively small sample size. We did not analyze other inflammation-related peripheral blood markers such prognostic nutritional index and Creactive protein-to-albumin ratio because not as many patients had data from liver function test and C-reactive protein [6,35]. In addition, our study population was obtained from a single center, which might limit generalizing the results to other countries or ethnicities. Future larger multi-center prospective studies would be useful in validating our findings. Lastly, although this is a case-control study involving multiple cancer types, the largest 
proportion of patients had lung cancer and this limits extrapolating the results to all cancer types as a whole. However, this study also has considerable strengths. This is the first study, to our knowledge, to employ a case-control study design to look at the association between NLR and the development of irAEs. First, as cases and controls were similar in terms of age, gender, and ethnicity, the effects of these variables as risk factors or confounders were not examined through use of logistic regression. Second, unlike other cross-sectional studies, the confounding effects of cancer stage and type on NLR and PLR were already accounted for during the study design stage. Third, our data was derived from multi-ethnic patients with different cancer types receiving various classes of ICIs, reflecting a real-world scenario. This makes demonstration of NLR to be an independent prognostic factor for irAEs, PFS, and OS in this heterogenous population all the more remarkable.

\section{Conclusions}

Our case-control study has demonstrated that NLR was independently associated with irAEs, PFS and OS. NLR has a bidirectional relationship with adverse outcomes. Lower NLR was associated with increased occurrence of irAEs while higher NLR values were associated with worse clinical outcomes. In addition, reduction of NLR at week 6 was associated with improved clinical outcomes. We propose that NLR is an inexpensive, readily available biomarker to predict different outcomes in cancer patients receiving ICI therapy. Prospective studies with large cohorts are warranted to validate the clinical application of our findings.

Supplementary Materials: The following are available online at https:/ /www.mdpi.com/2072-669 $4 / 13 / 6 / 1308 / s 1$, Table S1. Univariate binary logistic regression analysis to determine risk factors for irAEs; Table S2. Multivariate binary logistic regression analysis of factors associated with irAEs, Table S3. Univariate analyses of PFS and OS, Table S4. Studies on NLR and PLR in the development of irAEs, Table S5. Studies looking at the association of NLR and PLR with PFS and OS

Author Contributions: S.H.T., Y.H., A.S.C.W. and F.A. contributed to the conception or design of the work; P.Y.L., K.Q.X.O. and G.R.S.L. collected and curated the data; S.H.T. and Y.H.C. performed data analysis and interpretation; J.L.H., M.M., D.Q.H., F.S.W.T., A.Y.L., A.M., N.S.C., C.L.T., P.Y., E.S.T., K.W.P.N., J.V., Y.C.C., L.L.T., M.B.-H.L., H.R.C., W.Z.H., E.S.Y., D.K.L. and Y.S.Y. provided patient data for analysis; P.Y.L., K.Q.X.O., G.R.S.L. and S.H.T. drafted the manuscript; S.H.T. oversaw the project. All authors have read and agreed to the published version of the manuscript.

Funding: This work was supported by the following grant support: (i) Centre for Precision Health, National University Health System and (ii) National Research Foundation, Singapore and National Medical Research Council, Singapore under its NMRC Centre Grant Programme (NMRC/CG/M005/ 2017_NCIS).

Institutional Review Board Statement: The study was approved by NHG Domain Specific Review Board B (reference code: 2017/01254) and was carried out in accordance with the principles of the Declaration of Helsinki.

Informed Consent Statement: Informed consent was obtained from all subjects involved in the study.

Data Availability Statement: Data are not publicly available due to privacy and ethical restrictions.

Acknowledgments: The authors wish to thank Lelia Sze Yee Tay for the data collection and secretarial assistance.

Conflicts of Interest: The authors declare no conflict of interest.

\section{References}

1. Kohrt, H.E.; Tumeh, P.C.; Benson, D.; Bhardwaj, N.; Brody, J.; Formenti, S.; Fox, B.A.; Galon, J.; June, C.H.; Kalos, M.; et al. Immunodynamics: A cancer immunotherapy trials network review of immune monitoring in immuno-oncology clinical trials. J. Immunother. Cancer 2016, 4, 15. [CrossRef] 
2. Khan, M.; Lin, J.; Liao, G.; Tian, Y.; Liang, Y.; Li, R.; Liu, M.; Yuan, Y. Comparative analysis of immune checkpoint inhibitors and chemotherapy in the treatment of advanced non-small cell lung cancer: A meta-analysis of randomized controlled trials. Medicine 2018, 97, e11936. [CrossRef] [PubMed]

3. Suarez-Almazor, M.E.; Kim, S.T.; Abdel-Wahab, N.; Diab, A. Review: Immune-Related Adverse Events With Use of Checkpoint Inhibitors for Immunotherapy of Cancer. Arthritis Rheumatol. 2017, 69, 687-699. [CrossRef] [PubMed]

4. Koelzer, V.H.; Rothschild, S.I.; Zihler, D.; Wicki, A.; Willi, B.; Willi, N.; Voegeli, M.; Cathomas, G.; Zippelius, A.; Mertz, K.D. Systemic inflammation in a melanoma patient treated with immune checkpoint inhibitors-an autopsy study. J. Immunother. Cancer 2016, 4, 1-8. [CrossRef] [PubMed]

5. Diem, S.; Schmid, S.; Krapf, M.; Flatz, L.; Born, D.; Jochum, W.; Templeton, A.J.; Früh, M. Neutrophil-to-Lymphocyte ratio (NLR) and Platelet-to-Lymphocyte ratio (PLR) as prognostic markers in patients with non-small cell lung cancer (NSCLC) treated with nivolumab. Lung Cancer 2017, 111, 176-181. [CrossRef] [PubMed]

6. Peng, L.; Wang, Y.; Liu, F.; Qiu, X.; Zhang, X.; Fang, C.; Qian, X.; Li, Y. Peripheral blood markers predictive of outcome and immune-related adverse events in advanced non-small cell lung cancer treated with PD-1 inhibitors. Cancer Immunol. Immunother. 2020, 69, 1813-1822. [CrossRef]

7. Pavan, A.; Calvetti, L.; Dal Maso, A.; Attili, I.; Del Bianco, P.; Pasello, G.; Guarneri, V.; Aprile, G.; Conte, P.; Bonanno, L. Peripheral Blood Markers Identify Risk of Immune-Related Toxicity in Advanced Non-Small Cell Lung Cancer Treated with Immune-Checkpoint Inhibitors. Oncologist 2019, 24, 1128-1136. [CrossRef] [PubMed]

8. Robert, C. A decade of immune-checkpoint inhibitors in cancer therapy. Nat. Commun. 2020, 11, 3801. [CrossRef]

9. Ma, L.; Zeng, A.; Chen, B.; Chen, Y.; Zhou, R. Neutrophil to lymphocyte ratio and platelet to lymphocyte ratio in patients with systemic lupus erythematosus and their correlation with activity: A meta-analysis. Int. Immunopharmacol. 2019, 76 , 105949. [CrossRef]

10. Zhou, X.; Yao, Z.; Yang, H.; Liang, N.; Zhang, X.; Zhang, F. Are immune-related adverse events associated with the efficacy of immune checkpoint inhibitors in patients with cancer? A systematic review and meta-analysis. BMC Med. 2020, 18, 87. [CrossRef]

11. Zahorec, R. Ratio of neutrophil to lymphocyte counts-rapid and simple parameter of systemic inflammation and stress in critically ill. Bratisl. Lek. List. 2001, 102, 5-14.

12. Smith, R.A.; Bosonnet, L.; Ghaneh, P.; Sutton, R.; Evans, J.; Healey, P.; Garvey, C.; Hughes, M.; Raraty, M.; Campbell, F.; et al. The platelet-lymphocyte ratio improves the predictive value of serum CA19-9 levels in determining patient selection for staging laparoscopy in suspected periampullary cancer. Surgery 2008, 143, 658-666. [CrossRef]

13. Kim, S.; Eliot, M.; Koestler, D.C.; Wu, W.C.; Kelsey, K.T. Association of Neutrophil-to-Lymphocyte Ratio With Mortality and Cardiovascular Disease in the Jackson Heart Study and Modification by the Duffy Antigen Variant. JAMA Cardiol. 2018, 3, 455-462. [CrossRef] [PubMed]

14. Condamine, T.; Dominguez, G.A.; Youn, J.I.; Kossenkov, A.V.; Mony, S.; Alicea-Torres, K.; Tcyganov, E.; Hashimoto, A.; Nefedova, Y.; Lin, C.; et al. Lectin-type oxidized LDL receptor-1 distinguishes population of human polymorphonuclear myeloid-derived suppressor cells in cancer patients. Sci. Immunol. 2016, 1, aaf8943. [CrossRef] [PubMed]

15. Veglia, F.; Perego, M.; Gabrilovich, D. Myeloid-derived suppressor cells coming of age. Nat. Immunol. 2018, 19, 108-119. [CrossRef]

16. Tay, S.H.; Celhar, T.; Fairhurst, A.M. Low-Density Neutrophils in Systemic Lupus Erythematosus. Arthritis Rheumatol. 2020, 72, 1587-1595. [CrossRef]

17. Basu, A.; Kollengode, K.A.; Rafatnia, A.; Manoli, H.; Danenberg, G.; Chakravartty, E.; Epstein, A.L.; Pinski, J.K. Relationship between neutrophil lymphocyte ratio (NLR) and MDSC concentration in localized and metastatic castration resistant prostate cancer (mCRPC) patients. J. Clin. Oncol. 2018, 36, 338. [CrossRef]

18. Gasparyan, A.Y.; Ayvazyan, L.; Mukanova, U.; Yessirkepov, M.; Kitas, G.D. The Platelet-to-Lymphocyte Ratio as an Inflammatory Marker in Rheumatic Diseases. Ann. Lab. Med. 2019, 39, 345-357. [CrossRef]

19. Eun, Y.; Kim, I.Y.; Sun, J.M.; Lee, J.; Cha, H.S.; Koh, E.M.; Kim, H.; Lee, J. Risk factors for immune-related adverse events associated with anti-PD-1 pembrolizumab. Sci. Rep. 2019, 9, 14039. [CrossRef]

20. Nakamura, Y.; Tanaka, R.; Maruyama, H.; Ishitsuka, Y.; Okiyama, N.; Watanabe, R.; Fujimoto, M.; Fujisawa, Y. Correlation between blood cell count and outcome of melanoma patients treated with anti-PD-1 antibodies. Jpn. J. Clin. Oncol. 2019, 49, 431-437. [CrossRef]

21. Giannicola, R.; D’arrigo, G.; Botta, C.; Agostino, R.; Del Medico, P.; Falzea, A.C.; Barbieri, V.; Staropoli, N.; Del Giudice, T.; Pastina, P.; et al. Early blood rise in auto-antibodies to nuclear and smooth muscle antigens is predictive of prolonged survival and autoimmunity in metastatic-non-small cell lung cancer patients treated with PD-1 immune-check point blockade by nivolumab. Mol. Clin. Oncol. 2019, 11, 81-90. [CrossRef] [PubMed]

22. Das, S.; Johnson, D.B. Immune-related adverse events and anti-tumor efficacy of immune checkpoint inhibitors. J. Immunother. Cancer 2019, 7, 306. [CrossRef]

23. Owen, D.H.; Wei, L.; Bertino, E.M.; Edd, T.; Villalona-Calero, M.A.; He, K.; Shields, P.G.; Carbone, D.P.; Otterson, G.A. Incidence, Risk Factors, and Effect on Survival of Immune-related Adverse Events in Patients With Non-Small-cell Lung Cancer. Clin. Lung Cancer 2018, 19, e893-e900. [CrossRef] 
24. Lin, B.D.; Hottenga, J.J.; Abdellaoui, A.; Dolan, C.V.; de Geus, E.J.; Kluft, C.; Boomsma, D.I.; Willemsen, G. Causes of variation in the neutrophil-lymphocyte and platelet-lymphocyte ratios: A twin-family study. Biomark. Med. 2016, 10, 1061-1072. [CrossRef] [PubMed]

25. Prabawa, I.P.Y.; Bhargah, A.; Liwang, F.; Tandio, D.A.; Tandio, A.L.; Lestari, A.A.W.; Budiana, I.N.G.; Manuaba, I.B.A.P. Pretreatment Neutrophil-to-Lymphocyte ratio (NLR) and Platelet-to-Lymphocyte Ratio (PLR) as a Predictive Value of Hematological Markers in Cervical Cancer. Asian Pac. J. Cancer Prev. 2019, 20, 863-868. [CrossRef]

26. Guo, W.; Lu, X.; Liu, Q.; Zhang, T.; Li, P.; Qiao, W.; Deng, M. Prognostic value of neutrophil-to-lymphocyte ratio and plateletto-lymphocyte ratio for breast cancer patients: An updated meta-analysis of 17079 individuals. Cancer Med. 2019, 8, 4135-4148. [CrossRef]

27. Zhang, J.; Zhang, H.Y.; Li, J.; Shao, X.Y.; Zhang, C.X. The elevated NLR, PLR and PLT may predict the prognosis of patients with colorectal cancer: A systematic review and meta-analysis. Oncotarget 2017, 8, 68837-68846. [CrossRef] [PubMed]

28. Suh, K.J.; Kim, S.H.; Kim, Y.J.; Kim, M.; Keam, B.; Kim, T.M.; Kim, D.W.; Heo, D.S.; Lee, J.S. Post-treatment neutrophil-tolymphocyte ratio at week 6 is prognostic in patients with advanced non-small cell lung cancers treated with anti-PD-1 antibody. Cancer Immunol. Immunother. 2018, 67, 459-470. [CrossRef]

29. Nakaya, A.; Kurata, T.; Yoshioka, H.; Takeyasu, Y.; Niki, M.; Kibata, K.; Satsutani, N.; Ogata, M.; Miyara, T.; Nomura, S. Neutrophil-to-lymphocyte ratio as an early marker of outcomes in patients with advanced non-small-cell lung cancer treated with nivolumab. Int. J. Clin. Oncol. 2018, 23, 634-640. [CrossRef]

30. Fukui, T.; Okuma, Y.; Nakahara, Y.; Otani, S.; Igawa, S.; Katagiri, M.; Mitsufuji, H.; Kubota, M.; Hiyoshi, Y.; Ishihara, M.; et al. Activity of Nivolumab and Utility of Neutrophil-to-Lymphocyte Ratio as a Predictive Biomarker for Advanced Non-Small-Cell Lung Cancer: A Prospective Observational Study. Clin. Lung Cancer 2019, 20, 208-214.e2. [CrossRef]

31. Sagiv, J.Y.; Michaeli, J.; Assi, S.; Mishalian, I.; Kisos, H.; Levy, L.; Damti, P.; Lumbroso, D.; Polyansky, L.; Sionov, R.V.; et al. Phenotypic diversity and plasticity in circulating neutrophil subpopulations in cancer. Cell Rep. 2015, 10, 562-573. [CrossRef] [PubMed]

32. Gentles, A.J.; Newman, A.M.; Liu, C.L.; Bratman, S.V.; Feng, W.; Kim, D.; Nair, V.S.; Xu, Y.; Khuong, A.; Hoang, C.D.; et al. The prognostic landscape of genes and infiltrating immune cells across human cancers. Nat. Med. 2015, 21, 938-945. [CrossRef] [PubMed]

33. Gooden, M.J.; de Bock, G.H.; Leffers, N.; Daemen, T.; Nijman, H.W. The prognostic influence of tumour-infiltrating lymphocytes in cancer: A systematic review with meta-analysis. Br. J. Cancer 2011, 105, 93-103. [CrossRef]

34. de Coaña, Y.P.; Poschke, I.; Gentilcore, G.; Mao, Y.; Nyström, M.; Hansson, J.; Masucci, G.V.; Kiessling, R. Ipilimumab treatment results in an early decrease in the frequency of circulating granulocytic myeloid-derived suppressor cells as well as their Arginase1 production. Cancer Immunol. Res. 2013, 1, 158-162. [CrossRef]

35. Kondo, T.; Nomura, M.; Otsuka, A.; Nonomura, Y.; Kaku, Y.; Matsumoto, S.; Muto, M. Predicting marker for early progression in unresectable melanoma treated with nivolumab. Int. J. Clin. Oncol. 2019, 24, 323-327. [CrossRef] [PubMed] 